CIENCIAMATRIA

Revista Interdisciplinaria de Humanidades, Educación, Ciencia y Tecnología

Año VI. Vol. VI. N${ }^{\circ}$. Edición Especial. 2020

Hecho el depósito de ley: pp201602FA4721 ISSN-L: 2542-3029; ISSN: 2610-802X

Universidad Nacional Experimental Francisco de Miranda (UNEFM). Santa Ana de Coro. Venezuela

Shirley Fiorella Simbron-Espejo; Fanny Miriam Sanabria-Boudri

DOI $10.35381 / \mathrm{cm} . v 6 \mathrm{i} 10.295$

\title{
Liderazgo directivo, clima organizacional y satisfacción laboral del docente
}

\section{Leadership leadership, organizational climate and teacher job satisfaction}

\author{
Shirley Fiorella Simbron-Espejo \\ Fiorella24 acuario shirley@hotmail.com \\ Universidad Privada del Norte \\ Perú \\ https://orcid.org/0000-0002-7521-0315 \\ Fanny Miriam Sanabria-Boudri \\ fanny miriam@hotmail.com \\ Universidad Nacional Enrique Guzmán y Valle \\ Perú \\ https://orcid.org/0000-0002-2462-2715
}

Recibido: 20 de noviembre de 2019

Aprobado: 1 de diciembre de 2019

\begin{abstract}
RESUMEN
El objetivo de la investigación fue determinar la relación entre el liderazgo directivo, clima organizacional y la satisfacción laboral del docente en la universidad de Ciencias y Humanidades del distrito de los Olivos. El estudio tuvo un diseño descriptivo correlacional, la muestra quedó conformada por 35 docentes pertenecientes a la Facultad de Humanidades y Ciencias Sociales de la Universidad de Ciencias y Humanidades. Se utilizaron tres instrumentos diseñados específicamente para este estudio; uno sobre liderazgo del directivo, clima organizacional y la satisfacción laboral del docente. A partir de los datos analizados se concluye que existe una relación estadísticamente significativa entre el liderazgo directivo, clima organizacional y satisfacción laboral de los docentes de la Universidad de Ciencias y Humanidades del distrito de los Olivos, esta correlación es positiva alta entre los tres variables.
\end{abstract}

Descriptores: Liderazgo directivo; clima organizacional; satisfacción laboral; docente, universidad. 


\begin{abstract}
The objective of the research was to determine the relationship between managerial leadership, organizational climate and teacher job satisfaction at the University of Science and Humanities of the Olivos district. The study had a descriptive correlational design, the sample was made up of 35 teachers belonging to the Faculty of Humanities and Social Sciences of the University of Sciences and Humanities. Three instruments designed specifically for this study were used; one on managerial leadership, organizational climate and teacher job satisfaction. Based on the data analyzed, it is concluded that there is a statistically significant relationship between the managerial leadership, organizational climate and job satisfaction of teachers at the University of Science and Humanities of the Olivos district, this correlation is positive high among the three variables.
\end{abstract}

Descriptors: Managerial leadership; organizational climate; job satisfaction; teacher, university.

\title{
INTRODUCCIÓN
}

En una realidad sumamente competitiva, las organizaciones implementan constantemente diversas herramientas necesarias para mejorar el desempeño de su personal, con la finalidad de alcanzar un buen nivel de productividad, rentabilidad y lograr un posicionamiento empresarial expectante. De acuerdo con dicha premisa, para toda institución resulta importante medir y conocer su liderazgo, además del clima organizacional, porque así puede impactar significativamente en los resultados, al integrar los comportamientos y aptitudes hacia las metas comunes, marcando la diferencia entre una empresa de buen desempeño y otra de bajo desempeño.

De acuerdo con Zarate (2011), la mayoría de las personas están de acuerdo en afirmar que se encuentra ante una situación nueva, una época histórica en la que el cambio se ha convertido en el principal protagonista. En la actualidad el índice de cambio es más rápido, su magnitud es mucho mayor y la cantidad y calidad del mismo es diferente, lo que hace que los responsables de las instituciones educativas tengan que enfrentarse con problemas que nunca habían visto antes.

El liderazgo y la calidad educativa es un componente que abarca las formas a través de las cuales la educación permite satisfacer de una forma efectiva las necesidades y 
expectativas de la comunidad educativa en general (estudiantes, docentes, padres de familia, personal administrativo y de servicios, así como la comunidad en general) como entes involucrados en dicho proceso (Campos, 2012). En relación al clima organizacional, es posible considerarlo desde una visión psicológica como un estilo de comportamiento organizacional. El clima es una variable multifacético que influye en muchos aspectos de la vida organizacional.

Por ello, las organizaciones están llamadas a establecer estrategias que permitan observar el sinnúmero de variables que se generan dentro de su funcionamiento y que de una $u$ otra manera influyen, negativa o positivamente, al normal funcionamiento administrativo-productivo-interaccional que se establece dentro de esta. Dentro de este contexto, la productividad y el manejo del capital humano en las organizaciones se convierten los elementos claves de la subsistencia en el tiempo de estas, por lo que las variables que intervienen en estos procesos son relevantes al momento de realizar un diagnóstico organizacional. Entre ellos la satisfacción del trabajador ocupa un lugar preferente, pues la percepción negativa o positiva de los trabajadores influye en la relación que estos adopten con la organización y el nivel de compromiso y productividad que esta tenga.

Bajo estas premisas, se atraviesa por una crisis generalizada y en todos los ámbitos gerenciales, y el mundo educativo universitario no es la excepción. Las competencias están omnipresentes, más como retórica que como realidad, particularmente en los diseños curriculares y evaluaciones, están planteadas para un mundo que no existe debido a que los tiempos han cambiado, la tecnología ha revolucionado los estilos de vida de todo la gente y la universidad ya no debería ser lo mismo del siglo pasado.

En este caso, es fundamental, considerar el liderazgo de los directores para poder conducir y guiar a los miembros de una institución, en este caso la universidad, con el fin de cumplir los objetivos y metas planificadas. Debido a que tanto la gerencia como la administración buscan la eficacia y la efectividad que dé resultados para responder acertadamente en la solución de la problemática existen en diversas áreas de 
conocimiento y gestión.

Quien enfrenta el reto de dirigir una institución educativa, más que ser solo un administrador, tendrá necesariamente que ser un líder educativo, para lograr resultados óptimos en las condiciones en que se vive (Alfonso, 2001), no basta con reunir los requisitos necesarios para el cargo, ni acumular una larga trayectoria docente y directiva, sino también una visión que trascienda los parámetros de la institución educativa.

La nueva gestión en este siglo, (Gallegos, 2004) requiere que se haga las tareas administrativas y pedagógicas actuando de un modo $u$ otro de acuerdo al grado de confianza de sus colaboradores, prevaleciendo sobre los estilos burocráticos, donde lo pedagógico se ve afectado por decisiones y mecanismos de corte administrativo, lo cual parece ser que el gran "nudo" que se interpone entre la organización educativa y su eficiencia está en la ruptura de la gestión con la pedagogía, que existe en las escuelas de administración más tradicional, cuyo patrón se ajusta en mayor medida al control y la supervisión.

Toda esta problemática está relacionada con el ambiente laboral o clima organizacional, forma parte intrínseca de la organización. En este sentido, se considera que cualquier factor del ambiente laboral modifica un cambio directo en el clima organizacional (Centeno, 1999). Es un fenómeno interviniente que media entre los factores del sistema organizacional y las tendencias motivacionales que se traducen en un comportamiento que tiene consecuencias sobre la organización.

Sin embargo, no solo influye en el contexto de trabajo, sino influye en la satisfacción laboral misma de las personas. Según Robbins (1996, p.181) la satisfacción laboral es la actitud general de un individuo hacia su trabajo. Se concentra en los sentimientos afectivos frente al trabajo y a las consecuencias posibles que se derivan de él. Este mismo autor señala que los factores más importantes que conducen a la satisfacción en el trabajo son: un trabajo desafiante desde el punto de vista mental, recompensas equitativas, condiciones de trabajo que constituyen un respaldo, colegas que apoyen y 
el ajuste personalidad - puesto, entre otros.

La realidad particular es que pareciera que estas variables aplicadas en la realidad están ausentes. En el área de gestión del liderazgo no se ven resultados, presentan un nivel de gestión básico o ineficiente, donde el nivel más bajo de logro corresponde a conducción, dimensión que da cuenta de la debilidad del equipo directivo para coordinar las acciones y los actores de la Universidad en función del logro de objetivos institucionales. Esto afecta el clima y la satisfacción laboral tanto de docentes como administrativos.

Desde la perspectiva del clima denota la elaboración del concepto desde las propiedades y características percibidas de la organización, se trata de un fenómeno complejo y con múltiples niveles; por ejemplo, actualmente la bibliografía existente debate sobre dos tipos de clima: el psicológico y el organizacional. Y cuando el liderazgo es ausente, simplemente esto se ve afectada y la gestión institucional no funciona. En consecuencia, en el objetivo de la investigación fue Determinar la relación entre el liderazgo directivo, clima organizacional y la satisfacción laboral del docente en la universidad de Ciencias y Humanidades del distrito de los Olivos.

\section{REVISIÓN TEÓRICA}

\section{Liderazgo directivo}

El liderazgo directivo se dice de las personas o líderes que guían, enseñan tienen una manera particular de dirigir una institución u organización y ayudan a la obtención de buenos resultados. Es aquella persona que en principio solicita la opinión de sus subordinados y da instrucciones detalladas de cómo, cuándo y dónde deben llevar a cabo una tarea específica para solucionar un problema. Es un estilo adecuado cuando la escasez de tiempo impide explicar con detalle los asuntos; si el directivo líder, ha generado un clima de confianza, los subordinados asumirán que el directivo ha cambiado y es el adecuado, porque las circunstancias así lo requieren.

Asimismo, se comprende como la capacidad de una organización de lograr 
aprendizajes en todos sus estudiantes, sin exclusión de ninguno. De esta definición se puede deducir que el liderazgo es cualidad de la persona que lo ejerce y también puede constituirse en una característica de la gestión de la institución, en la que personas con liderazgo participan de un proceso liderado por el director, coordinando y contribuyendo al éxito de los resultados y metas de la organización.

A partir de esta particularidad se dice que: líder es aquella persona que es capaz de influir en el comportamiento de un grupo induciendo el desempeño necesario para la consecución de los objetivos (Jiménez, 2008). Ander - Egg (1997, p. 110) menciona que "líder en inglés es leader, derivado del verbo to lead (guiar). Vocablo de amplio uso en nuestra lengua, en la que se escribe y pronuncia de acuerdo con la fonética inglesa". Es la persona que tiene capacidad de persuadir o dirigir, derivado de sus cualidades personales, independientemente de su posición social, de sus cargos o funciones. Es el que tiene habilidad para conseguir adeptos y seguidores. Para algunos psicólogos sociales, la nota más característica del líder es la de ser el miembro de un grupo que más frecuente y persistentemente es percibido desempeñando actos de influencia sobre los otros miembros del grupo. Como consecuencia de sus atributos de personalidad y de sus habilidades expresadas en una situación contextual determinada.

\section{Liderazgo pedagógico directivo}

Los directivos deben desarrollar habilidades de tipo conceptual que le permitan percibir tanto el todo como las partes, de tipo humanístico para lograr adecuada cooperación de las personas y de carácter técnico relativo a las formas de trabajo, la cual no se dan en la institución educativa (fuentes, 2011).

Según Pozner (1995) toda institución educativa necesita un liderazgo directivo. Persona o personas que asuman la conducción de la organización en relación a las metas y objetivos. Esta forma de gestión puede visibilizarse en:

a) El ejercicio del liderazgo pedagógico, a fin de hacer transitar a la institución educativa hacia estándares superiores de enseñanza y posibilitar que se 


\section{Shirley Fiorella Simbron-Espejo; Fanny Miriam Sanabria-Boudri}

comprometan con los resultados.

b) La promoción de la organización autónoma de sus estudiantes para generar participación en la vida escolar.

c) Una organización es abierta, comunicativa y flexible, más democrática en sus procedimientos de toma de decisiones.

d) La institucionalización de la evolución como práctica de docente colectiva y del aprendizaje; es decir que toda la comunidad educativa aprenda a mirarse y a valorar la evolución como una oportunidad para aprender, tanto de los logros como de los errores.

De acuerdo a Bolívar (1997) el liderazgo pedagógico es un factor importante para el mejoramiento de la educación y es una prioridad en las agendas de las políticas educativas. En este sentido, es importante el concepto de liderazgo y su reconocimiento en la organización escolar. El ejercicio de la dirección como liderazgo se ve como una práctica distribuida, más democrática dispersa en el conjunto de la organización. Por otro lado, Murillo (2006) señala que el liderazgo pedagógico, en lugar de una dimensión transaccional, conlleva una labor transformadora, pues no se limita a trabajar en las condiciones existentes y con las metas dadas, sino que va alterando aquellas condiciones del centro y del aula para que mejoren la educación ofrecida y las prácticas docentes en el aula.

\section{Elementos del liderazgo educativo}

En este sentido, Gallegos (2004, p. 117) señala que, reconociendo al liderazgo como un proceso, se consideran los siguientes elementos:

a) Objetivo. Propósitos a alcanzar en lo institucional e individual. El líder debe tener claro la finalidad educativa, apelando a nexos comunes y buscando la unidad.

b) Poder. El liderazgo es una forma de ejercicio del poder. Las cualidades del líder permiten la adhesión voluntaria de los agentes educativos, cuando ven 
credibilidad e integridad en sus acciones.

c) Estilo. Conducta que manifiesta el líder en el ejercicio de su poder, para integrar intereses y lograr objetivos, evitando los extremos: la autocracia y la pusilanimidad, aspectos reñidos con el verdadero significado de liderazgo.

d) Seguidores. La esencia del liderazgo es la cohesión, subordinación, el seguimiento y la emulación de los integrantes del grupo. Se sigue a un líder cuando es correcto, es decir "cuando hace lo que dice y dice lo que hace".

Afirma Gallegos (2004) que el liderazgo es un proceso que abarca estos cuatro elementos ya que la presencia de ellos es importante para hablar de liderazgo:

El primero elemento (objetivo) tener los propósitos o metas establecidos de tal forma que el líder y los seguidores en la organización estar al corriente para trabajar hacia ellos.

El segundo elemento (poder) es una facultad que tiene el líder para conducir y guiar al grupo en una organización donde el uso de sus capacidades, habilidades y conocimientos del liderazgo permita influir en las personas de manera voluntaria. Ejercer el liderazgo es ejecutar el poder debido a la influencia, movilización, voluntades y acciones que logra el líder en su organización.

EI tercer elemento (estilo) es otro elemento del liderazgo donde se manifiesta en la conducta y forma de relacionarse del líder con el grupo; en el ejercicio de su poder está siempre presente el liderazgo, sea mostrando un estilo democrático, liberal o autoritario, veremos al líder asumiendo uno de estos estilos o combinando alguno de ellos.

EI cuarto elemento (los seguidores) elemento de liderazgo importante, ya que ellos son las personas del grupo que hay que movilizar, cohesionar y lograr influir para que sigan el camino que traza el líder que lógicamente para alcanzar las metas y/o objetivos. 


\section{Director y la calidad educativa}

Calero (1998, p. 215) señala que, el trabajo del director para el mejoramiento de la educación es la toma adecuada de decisiones. Decidir en forma acertada es un proceso tan importante como el proceso mismo, con esto quiere decir que para encontrar una excelencia educativa se requiere de múltiples factores: entre otros, de profesores efectivos, de condiciones pedagógicas buenas, de estudiantes en buenas condiciones físicas y psicológicas, de políticas educativas claras, de universos valorativos identificables, de fluidez financiera y administrativa a nivel macro y también de la existencia cotidiana de las decisiones que se toman. Las grandes políticas y los grandes planes se convierten en decisiones reales y hechos visibles, a través de sus decisiones. Un director es un educador no porque está involucrado directamente en el hecho pedagógico, sino porque a través de sus decisiones puede contribuir a hacerlo más efectivo, facilitándole al docente su tarea, garantizándole el funcionamiento efectivo de la organización y/o dándole sentido dentro del entorno social.

El mismo autor Calero (2005, p. 280) señala que ser director implica decidir en función de:

a) Crear condiciones favorables al hecho pedagógico.

b) Garantizar el funcionamiento eficiente de la administración.

c) Influir y negociar con el medio social los procesos, insumos e intereses que requiere una institución para avanzar hacia mejores niveles.

Estas funciones precisas mejor el trabajo del director en una Institución educativa:

1. En la Gestión pedagógica debe tomar decisiones que faciliten a los docentes desarrollar con satisfacción y eficiencia profesional el hecho pedagógico. Poder decidir acertadamente para crear condiciones favorables hacia el aspecto pedagógico, requiere que el director tenga una gran comprensión del fenómeno educativo en general y en particular: que variables o factores lo afectan positiva y negativamente, bajo qué condiciones se puede esperar resultados, instrumentos necesarios para la medición de la calidad 
pedagógica, etc.

2. En la gestión Administrativa tiene que tomar decisiones sobre los procesos administrativos e institucionales para garantizar que el servicio educativo ocurra de una forma sostenida, regulada y de acuerdo a normas y propósitos enunciados a nivel institucional. Poder decidir en este nivel, requiere una gran comprensión del centro educativo como una institución social. El colegio o la escuela no sólo es el lugar a donde los alumnos van a recibir clases, es un espacio social en donde se tramitan símbolos, valores, lenguajes y relaciones específicas. Es también el lugar en donde la sociedad hace legítimos y válidos los saberes que juzga, es una parte fundamental del currículo. Los alumnos no aprenden solamente de lo que ocurre en la clase; aprenden también de la forma como discurre diariamente toda la institución escolar. Por eso las decisiones sobre la forma de definir y concebir el espacio físico, los horarios, la disciplina, los símbolos escolares, la forma como deben relacionarse los profesores con los alumnos y los profesores entre sí, la forma y el tipo de celebraciones, fiestas, etc.; son decisiones que un director debe pensar cuidadosamente porque todas ellas tienen implicancias educativas.

3. En la Gestión Institucional, el director decide, de alguna manera, sobre la institución como un todo. Debe tomar decisiones para buscar relacionar la institución de la mejor forma posible con la comunidad social: Con los padres de familia, con el gobierno, la parroquia, las personas e instituciones educativas. Este tipo de decisiones requiere, por parte del director, un gran conocimiento del entorno social, de las expectativas e intereses de otras instituciones, de las limitaciones y posibilidades tanto de la institución que dirige como de los grupos y entidades con los cuales se debe relacionar. Debe tomar decisiones para garantizar que la institución educativa se adecue y responda a las necesidades y expectativas de las comunidades que 
atiende en las que está ubicada.

\section{Clima organizacional}

Inicialmente los estudios de Clima y Cultura se hicieron independientemente, fue sólo en las últimas décadas que han sido discutidos simultáneamente en la literatura organizacional. La cultura se define como las creencias normativas (es decir, los valores del sistema) y comparte las expectativas de conducta (es decir, las normas del sistema) en una organización. Las normas del sistema se refieren explícitamente al sistema de conductas que deben considerarse entre los miembros de la Organización. El sistema de valores proporciona apoyo a las ideologías y justifica las conductas normativas y las actividades que se derivan de estas conductas. El sistema de valores y normas son a menudo vistos como los productos de la dinámica de grupo.

El clima psicológico es la percepción individual del empleado del impacto psicológico que genera el ambiente de trabajo sobre su bienestar. Además, cuando los empleados de un área particular de trabajo están de acuerdo sobre sus percepciones del ambiente de trabajo, estas percepciones compartidas se conjugan para describir el clima organizacional. El clima representa la personalidad de una organización y podría definirse como la percepción que los trabajadores se forman de la organización a la que pertenecen y que incide directamente en el desempeño de la organización; describe un grupo de características que muestra una organización o parte de ella.

A pesar de esta globalidad del concepto de clima, y a pesar de haber surgido a partir de una compresión de la organización como un sistema abierto, el clima de una organización es entendido habitualmente como medio interno, vale decir, en él se pone atención a variables y factores internos de la organización y no a los factores del entorno en que la organización se encuentra inmersa. Por otra parte, a pesar que el estudio del clima organizacional se encuentra enfocado a la comprensión de las variables ambientales internas que afectan el comportamiento de los individuos en la organización, su aproximación a estas variables es a través de la percepción que los 
individuos tienen de ellas.

La especial importancia de este enfoque reside en el hecho de que el comportamiento de un trabajador no es una resultante de los factores organizacionales existentes, sino que depende de las percepciones que tenga el trabajador de estos factores. Sin embargo, estas percepciones dependen de buena medida de las actividades, interacciones y otra serie de experiencias que cada miembro tenga con la empresa. De ahí que el Clima Organizacional refleja la interacción entre características personales y organizacionales.

Los factores y estructuras del sistema organizacional dan lugar a un determinado clima, en función a las percepciones de los miembros. Este clima resultante induce determinados comportamientos en los individuos. Estos comportamientos inciden en la organización y, por ende, en el clima, completando el circuito. Las variables consideradas en el concepto de clima organizacional son:

Variables del ambiente físico, tales como espacio físico, condiciones de ruido, calor, contaminación, instalaciones, maquinas, etc.

Variables estructurales, tales como tamaño de la organización, estructura formal, estilo de dirección, etc.

Variables del ambiente social, tales como compañerismo, conflictos entre personas o entre departamentos, comunicaciones, etc.

Variables personales, tales como aptitudes, actitudes, motivaciones, expectativas, etc.

Variables propias del comportamiento organizacional, tales como productividad, ausentismo, rotación, satisfacción laboral, tensiones y estrés, etc.

Todas estas variables configurarán el clima de una organización, a través de la percepción que de ellas tienen los miembros de la misma. Estas características de la organización son relativamente permanentes en el tiempo, se diferencian de una organización a otra y de una sección a otra dentro de una misma empresa. El Clima, junto con las estructuras y características organizacionales y los individuos que la componen, forman un sistema interdependiente altamente dinámico. Las percepciones 
y respuestas que abarcan el Clima Organizacional se originan en una gran variedad de factores.

Unos abarcan los factores de liderazgo y prácticas de dirección (tipos de supervisión: autoritaria, participativa, etc.). Otros factores están relacionados con el sistema formal y la estructura de la organización (sistema de comunicaciones, relaciones de dependencia, promociones, remuneraciones, etc.). Otros son las consecuencias del comportamiento en el trabajo (sistemas de incentivo, apoyo social, interacción con los demás miembros, etc.). El concepto de clima organizacional, en consecuencia, se refiere a las percepciones compartidas por los miembros de una organización respecto al trabajo, el ambiente físico en que éste se da, las relaciones interpersonales que tienen lugar en torno a él y las diversas regulaciones formales que afectan a dicho trabajo.

Se relaciona, también, con el concepto de Salud Mental de los individuos, entendido como la capacidad de una persona para sentirse bien consigo misma, respecto a los demás, y ser capaz de enfrentar por sí misma las exigencias de la vida. Finalmente, se define como la cualidad o propiedad del ambiente organizacional que experimentan los miembros de la organización e influye en su comportamiento. Como se puede ver, el concepto habitual de clima dice referencia con variables situacionales, pero medidas por las percepciones de los miembros de la organización. Dada nuestra definición de la organización como un sistema autopoiético de decisiones, los miembros de la organización no constituyen los elementos del sistema organizacional, sino parte de su ambiente: su ambiente interno.

El clima, en esta perspectiva, ha de ser entendido como el conjunto de apreciaciones que los miembros de la organización tienen de su experiencia en y con el sistema organizacional. Estas apreciaciones constituyen una afirmación evaluativa, una explicación valorativa de la experiencia organizacional de los miembros del sistema. En base a las consideraciones precedentes podríamos llegar a la siguiente definición de Clima Organizacional: El Clima Organizacional es un fenómeno interviniente que media 
entre los factores del sistema organizacional y las tendencias motivacionales que se traducen en un comportamiento que tiene consecuencias sobre la organización (productividad, satisfacción, rotación, etc.).

\section{Características del clima organizacional}

El clima organizacional implica una referencia constante de los miembros respecto a su estar en la organización. El sistema organizacional, como sistema autopoético de decisiones, constituye el tema de reflexiones sobre el que se construye la definición de clima. En otras palabras, el clima puede construirse como una autorreflexión de la organización acerca de su devenir. La experiencia organizacional que tienen los miembros es autoobservada por éstos, que la evalúan colectivamente.

Esto significa que el clima organizacional es una autoreflexión de los miembros de la organización acerca de su vinculación entre sí y con el sistema organizacional. Sin embargo, esto no hace que el clima sea necesariamente una autorreflexión de la organización como sistema autopoético de decisiones. Para que lo sea, es necesario que el tema del clima sea tratado en el decidir organizacional. En efecto, en los casos en que las organizaciones ven afectado su decidir por el clima o en los casos en que se adoptan decisiones, motivadas por el clima o destinadas a provocar cambios en éste, el clima ha pasado a constituirse en parte de la autorreflexión del sistema organizacional. El clima organizacional tiene las siguientes características:

a) Dice referencia con la situación en que tiene lugar el trabajo de la organización.

b) Las variables que definen el clima son aspectos que guardan relación con el ambiente laboral.

c) Tiene una cierta permanencia, a pesar de experimentar cambios por situaciones coyunturales. Esto significa que se puede contar con una cierta estabilidad en el clima de una organización, con cambios relativamente graduales, pero esta estabilidad puede sufrir perturbaciones de importancia derivadas de decisiones que afecten en forma relevante el devenir organizacional. Una situación de 
conflicto de no resuelto, por ejemplo, puede empeorar el clima organizacional por un tiempo comparativamente extenso.

d) Tiene un cierto impacto sobre los comportamientos de los miembros de la empresa. Un clima malo, por otra parte, hará extremadamente difícil la conducción de la organización y la coordinación de las labores.

e) Afecta el grado de compromiso e identificación de los miembros de la organización con ésta. Una organización con un buen clima tiene una alta probabilidad de conseguir un nivel significativo de identificación de sus miembros: en tanto, una organización cuyo clima sea deficiente no podrá esperar un alto grado de identificación. Las organizaciones que se quejan porque sus trabajadores " no tienen la camiseta puesta ", normalmente tienen un muy mal clima organizacional.

f) Es afectado por los comportamientos y actitudes de los miembros de la organización y, a su vez, afecta dichos comportamientos y actitudes. En otras palabras, un individuo puede ver cómo el clima de su organización es grato y sin darse cuenta- contribuir con su propio comportamiento a que este clima sea agradable; en el caso contrario, a menudo sucede que personas pertenecientes a una organización hacen amargas críticas al clima de sus organizaciones, sin percibir que con sus actitudes negativas están configurando este clima de insatisfacción y descontento.

g) Es afectado por diferentes variables estructurales, como estilo de dirección, políticas y planes de gestión, sistemas de contratación y despidos, etc. Estas variables, a su vez, pueden ser también afectadas por el clima. Por ejemplo, un estilo de gestión muy burocrático y autoritario, con exceso de control y falta de confianza en los subordinados puede llevar a un clima laboral tenso, de desconfianza y con actitudes escapistas e irresponsables de parte de los subordinados, lo que conducirá a un refuerzo del estilo controlador, autoritario y desconfiado de la jerarquía burocrática de la organización. Este es un círculo 


\section{Shirley Fiorella Simbron-Espejo; Fanny Miriam Sanabria-Boudri}

vicioso del que resulta difícil salir, porque el clima y el estilo de dirección se esfuerzan mutuamente en una escalada que cuesta romper, por cuanto para hacerlo sería necesario actuar en un sentido inverso a lo que el sistema de variables estilo de dirección -clima organizacional perecería requerir.

h) El ausentismo y la rotación excesiva puede ser indicaciones de un mal clima laboral. Algo semejante ocurre con la insatisfacción laboral desmejorado. La forma de atacar estos problemas, por lo tanto, puede ser difícil, dado que implica realizar modificaciones en el complejo de variables que configura el clima organizacional.

i) En estrecha conexión con lo anterior, es necesario señalar que el cambio en el clima organizacional es siempre posible, pero que se requiere de cambios en más de una variable para que el cambio sea duradero, es decir, para conseguir que el clima de la organización se estabilice en una nueva configuración.

En otras palabras, aunque es relativamente sencillo obtener cambios dramáticos y notarios en el clima organizacional mediante políticas o decisiones efectivas, es difícil lograr que el clima no vuelva a su situación anterior una vez que se han disipado los afectos de las medidas adoptadas. Por ejemplo, en una organización cuyo clima se caracteriza por el descontento generalizado, es posible lograr una mejora importante, pero pasajera, mediante la promesa de un aumento de remuneraciones.

El efecto de este anuncio, no obstante, pronto, será olvidado si no llevan a cabo otros cambios que permitan llevar a la organización a otra situación, a una diferente configuración del clima. Incluso es frecuente el caso en que un anuncio de mejoramientos de las remuneraciones, instalaciones físicas, relaciones laborales, etc., provoca expectativas y mejorías en el clima organizacional, para luego, ante la concreción efectiva de los cambios anunciados, volver a un clima organizacional que puede ser peor que inicial, debido a que los cambios anunciados generaron expectativas que no pudieron satisfacer. En este caso, se producen frustraciones, desconfianza y una actitud desesperanzada y altamente negativa hacia la organización, 
con el correspondiente efecto sobre el clima organizacional.

\section{Definición de Satisfacción Laboral}

La satisfacción en el trabajo puede ser definida como una actitud general del individuo hacia su trabajo. Para muchos la satisfacción en el trabajo es un motivo en sí mismo, es decir, el trabajador mantiene una actitud positiva en la organización laboral para lograr ésta. Para otros, es una expresión de una necesidad que puede o no ser satisfecha. En la medida en que la motivación actúe a fin de satisfacer las necesidades, produce como consecuencia en el ámbito laboral, la Satisfacción Laboral, término que podemos conceptuarlo como:

Resultado de diversas actitudes que tienen los trabajadores en relación al salario, la supervisión, el reconocimiento, oportunidades de ascenso (entre otros) ligados a otros factores como la edad, la salud, relaciones familiares, posición social, recreación y demás actividades en organizaciones laborales, políticas y sociales. El mencionado concepto está centrado en la satisfacción de las necesidades dependiendo de ciertos y determinados factores laborales, pero siempre tomando en alta importancia a que la satisfacción se logra a través de los diversos factores motivacionales, de ahí se desprende que el bienestar laboral se descompone en dos columnas básicas: la satisfacción de las necesidades y las aspiraciones de los individuos, ambos en relación con el trabajo.

\section{Importancia de la satisfacción laboral}

El tema de la satisfacción laboral es de gran interés porque nos indica la habilidad de la organización para satisfacer las necesidades de los trabajadores y además, por los siguientes motivos:

a) Existen muchas evidencias de que los trabajadores insatisfechos faltan a trabajar con más frecuencia y suelen renunciar más.

b) Se ha demostrado que los empleados satisfechos gozan de mejor salud y 
viven más años.

c) La satisfacción laboral se refleja en la vida particular del empleado.

Además, la satisfacción laboral es de gran interés en los últimos años por constituirse en resultados organizacionales que expresan el grado de eficacia, eficiencia y efectividad alcanzado por la institución; como tal, son indicadores del comportamiento de los que pueden derivar políticas y decisiones institucionales. Es más probable que los empleados satisfechos sean ciudadanos satisfechos, estas personas adoptarán una actitud más positiva ante la vida en general y representarán para la sociedad personas más sanas, en términos psicológicos. Actualmente se presta mayor interés a la calidad de vida laboral a diferencia de años anteriores en donde se buscaba la relación con el rendimiento. Subyace la idea de que las personas trabajen bien, pero sintiéndose bien; o al a inversa, que estén a gusto en el trabajo, al tiempo que ofrecen un resultado satisfactorio.

Es un hecho observable que los trabajadores que se sienten satisfechos en su trabajo, sea porque se consideran bien pagados 0 bien tratados, sea porque ascienden 0 aprenden, son quienes producen y rinden más. A la inversa, los trabajadores que se sienten mal pagados, maltratados, atascados en tareas monótonas, sin posibilidades de ampliar horizontes de comprensión de su labor, son los que rinden menos, es decir, son los más improductivos.

Todos somos capaces de percibir claramente lo benéfico, agradable y estimulante de estar en el trabajo con un grupo de personas que se llevan bien, que se comprenden, que se comunican, que se respetan, trabajan en armonía y cooperación. La buena atmósfera en el trato es indispensable para lograr un elevado rendimiento individual y colectivo de un grupo humano de trabajo, como lo es el oxígeno, para el normal funcionamiento de los pulmones y la respiración, lo cual se logra más que nada por una labor consiente de los jefes. (Atalaya, 1999, p.50). 


\section{CIENCIAMATRIA}

Revista Interdisciplinaria de Humanidades, Educación, Ciencia y Tecnología

Año VI. Vol. VI. N${ }^{\circ}$. Edición Especial. 2020

Hecho el depósito de ley: pp201602FA4721 ISSN-L: 2542-3029; ISSN: 2610-802X

Universidad Nacional Experimental Francisco de Miranda (UNEFM). Santa Ana de Coro. Venezuela

Shirley Fiorella Simbron-Espejo; Fanny Miriam Sanabria-Boudri

\section{MÉTODO}

El estudio asumió un enfoque cuantitativo. En este enfoque de investigación se acostumbra asociarlo con las técnicas estadísticas y la medición, con el acto de asignarle un valor a un dato, proceso o actividad de acuerdo con un conjunto de reglas, escala, niveles o patrones. Asimismo, el diseño de investigación fue no experimental, bajo un tipo de investigación correlacional, que según Hernández, Fernández y Baptista (2014), en este se trata de establecer la existencia de asociaciones significativas entre las variables liderazgo directivo, clima organizacional y satisfacción laboral.

La población y muestra quedo definida por 35 docentes pertenecientes a la Facultad de Humanidades y Ciencias Sociales de la Universidad de Ciencias y Humanidades del distrito de los Olivos. El análisis estadístico se realizó mediante la aplicación de técnicas estadísticas descriptivas y de correlación. En la encuesta de clima organizacional, satisfacción laboral y liderazgo directivo se determinó la relación entre las variables mediante la prueba de Pearson. 
CIENCIAMATRIA

Revista Interdisciplinaria de Humanidades, Educación, Ciencia y Tecnología

Año VI. Vol. VI. N${ }^{\circ}$. Edición Especial. 2020

Hecho el depósito de ley: pp201602FA4721 ISSN-L: 2542-3029; ISSN: 2610-802X

Universidad Nacional Experimental Francisco de Miranda (UNEFM). Santa Ana de Coro. Venezuela

Shirley Fiorella Simbron-Espejo; Fanny Miriam Sanabria-Boudri

\section{RESULTADOS}

\begin{tabular}{|c|c|c|c|c|}
\hline & & $\begin{array}{l}\text { Clima } \\
\text { organizacional }\end{array}$ & $\begin{array}{l}\text { Satisfacción } \\
\text { Laboral }\end{array}$ & $\begin{array}{l}\text { Liderazgo } \\
\text { Directivo }\end{array}$ \\
\hline \multirow[t]{3}{*}{$\begin{array}{l}\text { Clima } \\
\text { organizacional }\end{array}$} & $\begin{array}{l}\text { Correlación } \\
\text { de Pearson }\end{array}$ & 1 &, $902\left({ }^{* \star}\right)$ &, $832\left({ }^{* \star}\right)$ \\
\hline & $\begin{array}{l}\text { Sig, } \\
\text { (bilateral) }\end{array}$ & & ,000 & ,000 \\
\hline & $\mathrm{N}$ & 35 & 35 & 35 \\
\hline \multirow[t]{3}{*}{$\begin{array}{l}\text { Satisfacción } \\
\text { Laboral }\end{array}$} & $\begin{array}{l}\text { Correlación } \\
\text { de Pearson }\end{array}$ &, $902\left({ }^{* *}\right)$ & 1 &, $834\left({ }^{* *}\right)$ \\
\hline & $\begin{array}{l}\text { Sig, } \\
\text { (bilateral) }\end{array}$ & ,000 & & ,000 \\
\hline & $\mathrm{N}$ & 35 & 35 & 35 \\
\hline \multirow[t]{3}{*}{$\begin{array}{l}\text { Liderazgo } \\
\text { Directivo }\end{array}$} & $\begin{array}{l}\text { Correlación } \\
\text { de Pearson }\end{array}$ &, $832\left({ }^{* *}\right)$ &, $834(* *)$ & 1 \\
\hline & $\begin{array}{l}\text { Sig, } \\
\text { (bilateral) }\end{array}$ & ,000 & ,000 & \\
\hline & $\mathrm{N}$ & 35 & 35 & 35 \\
\hline
\end{tabular}

De acuerdo a la tabla mostrada, el coeficiente de correlación de Pearson $r=0,832$ para el liderazgo directivo y clima organizacional, $r=0,834$ para liderazgo directivo y satisfacción laboral, $r=0,902$ para clima organizacional y satisfacción laboral. Entonces existe una relación estadísticamente significativa entre el liderazgo directivo y clima organizacional en la satisfacción laboral del docente en la universidad de Ciencias y Humanidades del distrito de los Olivos, según el cuadro de Hernández, Fernández y Baptista (2010) es correlación positiva alta para liderazgo directivo y clima organizacional, es correlación positiva alta para liderazgo directivo y satisfacción laboral, es correlación positiva muy alta de clima organizacional y satisfacción laboral. 


\section{CONCLUSIONES}

En atención a la correlación liderazgo directivo y clima organizacional, los resultados concuerda con los hallazgos de Campos (2012), quien realizó una investigación con 100 docentes, padres de familia y estudiantes de la región Callao, evidenciando que existe una relación significativa entre el liderazgo directivo y cada una de sus dimensiones con el clima organizacional. Estos resultados se deben a que el director como líder logra persuadir y convencer a los miembros de la comunidad educativa para que los planes institucionales se lleven a cabo, tratando de superar dificultades y riesgos a los que se tenga que enfrentar (Espinoza, 1999), demostrando en esos momentos, poseer una habilidad para influir en los docentes, impactando en forma significativa para lograr resultados.

Bajo esta percepción los participantes consideran que el director posee la capacidad de ejercer influencia interpersonal a través del proceso comunicacional para lograr la consecución de uno o diversos objetivos específicos (Chiavenato, 1993) también se les compromete en la ejecución de un proceso mediante el cual un miembro del grupo (su líder) en este caso el director, influye a los otros miembros de la comunidad educativa hacia el logro de objetivos específicos grupales. En tal sentido, cabe notar que el liderazgo ejercido es eficaz, en la medida que logra manejar en diferente magnitud y flexibilidad los tres estilos estudiados (Blanchard, 2007).

Asimismo, existe una relación estadísticamente significativa entre las variables de liderazgo directivo, y la satisfacción laboral en los docentes. En cuanto al liderazgo directivo va tener influencia positiva o negativa en el clima y también en la satisfacción de los docentes. Estas debilidades son expresadas en un liderazgo directivo disperso, escasa autonomía y reconocimiento por el trabajo bien realizado y ausencia de evaluaciones que incidan en aspectos administrativos, en el mejoramiento cualitativo y cuantitativo y el mejoramiento de la educación en general (Lombardi, 2004). Navarro (2006) hace referencia a partir de su investigación, que los docentes al tener dominio sobre los contenidos que imparten, y cuando tienen una integridad, responsabilidad en 
su trabajo y mantienen y trabajo inequívoco con los valores de la institución, tienen o les generan mayor satisfacción labora.

Por ello recomienda que la universidad debe mejorar su clima organizacional y para ello debe formar personas para un mundo laboral donde las tareas a llevar a cabo estarán en constante evolución, el trabajo dependiente y mecánico cederá lugar al autónomo y profesional, reconocimiento a la labor desempeñada, De ahí, que el empleo de estrategias pedagógicas y académicas orientará a la formación progresiva del nuevo capital humano integra que se requiere para el desarrollo de un país. En atención a la relación estadísticamente significativa entre el clima organizacional y la satisfacción laboral del docente, según el cuadro de Hernández, Fernández y Baptista (2010) es correlación positiva muy alta.

\section{REFERENCIAS CONSULTADAS}

1. Alfonso, A. (2002). La excelencia gerencial en educación, Lima: Derrama Magisterial, Pág, 13, (B, Publica de Lima), C 371,1 E.

2. Atalaya, M. (1999). Satisfacción laboral y productividad. Revista de la Facultad de Psicología de la Universidad Mayor de San Marcos, 5, 45-76. Recuperado el 3 de noviembre de 2005, de la base de datos Emerald.

3. Bolívar, A. (1997). Liderazgo, mejora y centros educativos. En A. Medina (coord.): El liderazgo en educación. (pp. 25-46). Madrid: UNED.

4. Calero, M. (1998). Hacia la excelencia de la educación, Lima: San Marcos, Pág, 306, 215, (B,N,P,), 379,1 C23 1998

5. Calero, M. (2005). Hacia la Excelencia de la Educación. Lima: San Marcos. Pag. 306, 215. (B.N.P.). 379.1 C23.

6. Calero, M. (2006). Gestión Educativa. Lima: Abedul. Pág. 284, 30, 280. (BP).

7. Centeno, D. (1999). La comunicación oral y su influencia en el clima organizacional en una institución bancaria, Tesis inédita, Universidad Rafael Landívar, Guatemala. 
8. Chiavenato, I. (1993). Administración de recursos humanos (1e edición). McGraw Hill. Interamericana. México.

9. Chiavenato, I. (2006). Introducción a la teoría general de la administración. McGraw Hill. Interamericana. México.

10. Espinoza, M. (1999). Líderes del Tercer Milenio, Lima: EDIAS.

11. Herzberg, F. (1968). One More Time: How do you motivate employers? Harvard Business Review, Ja- Fe.

12. Hernández R., Fernández, C. y Baptista, P. (2014). Metodología de la Investigación. (6a. ed.). México: McGraw-Hill.

13. Jiménez, J. (2008). El valor de los valores en las organizaciones, Caracas: Cograf Ediciones.

14. Fuentes, L. (2011). Incidencia de la satisfacción laboral docente y el ambiente de aula en el rendimiento académico de los estudiantes universitarios. Tesis Doctoral. Universidad de Sevilla.

15. Gallegos, A. (2004). Gestión educativa en el proceso de descentralización, Lima: San Marcos, Pág, 112, 114, 117 (B, Publica de Lima).371,2 G15.

16. Lombardi, J. (2004). Comportamiento del clima organizacional en la facultad de agronomía de la universidad de Zulia, trabajo de grado. Universidad de Zulia.

17. Murillo, F. (2006). Una Dirección Escolar para el Cambio: del Liderazgo Transformacional al Liderazgo Distribuido. Revista Electrónica Iberoamericana sobre Calidad, Eficacia y Cambio en Educación, 4(4e). pp. 11-24. http://www.rinace.net/arts/vol4num4e/art2.pdf. Consultado el (Fecha).

18. Rivas, V. (2006). Clima organizacional y satisfacción laboral del docente de las escuelas zuliana de avanzada (Tesis de grado). Universidad Rafael Urdaneta, Maracaibo, Venezuela, Recuperado de http://200,35,84,131/portal/bases/marc/texto/9217-06-00064,pdf

19. Robbins, S. (1996). Comportamiento Organizacional. Edición Pearson Education. México D.F. 


\section{CIENCIAMATRIA}

Revista Interdisciplinaria de Humanidades, Educación, Ciencia y Tecnología

Año VI. Vol. VI. N${ }^{\circ}$. Edición Especial. 2020

Hecho el depósito de ley: pp201602FA4721 ISSN-L: 2542-3029; ISSN: 2610-802X

Universidad Nacional Experimental Francisco de Miranda (UNEFM). Santa Ana de Coro. Venezuela

Shirley Fiorella Simbron-Espejo; Fanny Miriam Sanabria-Boudri

20.Pozner, P. (1995). El directivo como gestor de los aprendizajes escolares. Aique: Buenos Aires.

21.Zarate, D. (2011). Liderazgo directivo y el desempeño docente en instituciones educativas de primaria del distrito de Independencia, Lima (Tesis de Maestría). Universidad Nacional Mayor De San Marcos, Lima, Perú.

\section{REFERENCES CONSULTED}

1. Alfonso, A. (2002). Management excellence in education, Lima: Master's Degree, Pág, 13, (B, Publica de Lima), C 371.1 E.

2. Atalaya, M. (1999). Job satisfaction and productivity. Journal of the Faculty of Psychology of the Universidad Mayor de San Marcos, 5, 45-76. Retrieved on November 3, 2005, from the Emerald database.

3. Bolívar, A. (1997). Leadership, improvement and educational centers. In A. Medina (coord.): Leadership in education. (pp. 25-46). Madrid: UNED.

4. Calero, M. (1998). Towards the excellence of education, Lima: San Marcos, Pág, 306, 215, (B, N, P, ), 379.1 C23 1998

5. Calero, M. (2005). Towards the Excellence of Education. Lima: San Marcos. Pag. 306, 215. (B.N.P.). 379.1 C23.

6. Calero, M. (2006). Education Management. Lima: Birch. P. 284, 30, 280. (BP).

7. Centeno, D. (1999). Oral communication and its influence on the organizational climate in a banking institution, unpublished thesis, Rafael Landívar University, Guatemala.

8. Chiavenato, I. (1993). Human resources administration (1st edition). McGraw Hill Interamerican Mexico.

9. Chiavenato, I. (2006). Introduction to the general theory of administration. McGraw Hill Interamerican Mexico.

10. Espinoza, M. (1999). Leaders of the Third Millennium, Lima: EDIAS,

11. Herzberg, F. (1968). One More Time: How do you motivate employers? Harvard Business Review, Jafe. 


\section{CIENCIAMATRIA}

Revista Interdisciplinaria de Humanidades, Educación, Ciencia y Tecnología

Año VI. Vol. VI. N${ }^{\circ}$. Edición Especial. 2020

Hecho el depósito de ley: pp201602FA4721 ISSN-L: 2542-3029; ISSN: 2610-802X

Universidad Nacional Experimental Francisco de Miranda (UNEFM). Santa Ana de Coro. Venezuela

Shirley Fiorella Simbron-Espejo; Fanny Miriam Sanabria-Boudri

12. Hernández R., Fernández, C. and Baptista, P. (2014). Investigation methodology. (6th ed.). Mexico: McGraw-Hill.

13. Jiménez, J. (2008). The value of values in organizations, Caracas: Cograf Ediciones.

14. Fuentes, L. (2011). Incidence of teacher job satisfaction and the classroom environment in the academic performance of university students. Doctoral thesis. Sevilla University.

15.Gallegos, A. (2004). Educational management in the decentralization process, Lima: San Marcos, Pág, 112, 114, 117 (B, Publica de Lima) .371.2 G15.

16. Lombardi, J. (2004). Organizational climate behavior in the agronomy faculty of the University of Zulia, undergraduate work. University of Zulia

17. Murillo, F. (2006). A School Office for Change: from Transformational Leadership to Distributed Leadership. Ibero-American Electronic Magazine on Quality, Efficiency and Change in Education, 4 (4e). pp. 11-24. http://www.rinace.net/arts/vol4num4e/art2.pdf. Consulted on (Date).

18. Rivas, V. (2006). Organizational climate and job satisfaction of the teacher of the Zuliana advanced schools (Thesis). Rafael Urdaneta University, Maracaibo, Venezuela, Retrieved from http: // 200,35,84,131 / portal / bases / marc / text / 9217-06-00064, pdf

19. Robbins, S. (1996). Organizational behavior. Pearson Education Edition. Mexico DF.

20. Pozner, P. (1995). The manager as a manager of school learning. Aique: Buenos Aires.

21.Zarate, D. (2011). Leadership leadership and teaching performance in primary educational institutions of the district of Independencia, Lima (Master's Thesis). National University of San Marcos, Lima, Peru. 